\title{
Convective Transport of Nanofluid Saturated with Porous Layer
}

\section{Jada Prathap Kumar, Jawali Channabasappa Umavathi, Channakeshava Murthy}

Department of Mathematics, Gulbarga University, Kalaburagi, Karnataka, India

Email address:

p_rathap@yahoo.com (J. P. Kumar)

\section{To cite this article:}

Jada Prathap Kumar, Jawali Channabasappa Umavathi, Channakeshava Murthy. Convective Transport of Nanofluid Saturated with Porous Layer. American Journal of Applied Mathematics. Vol. 5, No. 1, 2017, pp. 1-13. doi: 10.11648/j.ajam.20170501.11

Received: November 10, 2016; Accepted: November 26, 2016; Published: January 18, 2017

\begin{abstract}
In the present article, the onset of convection in a horizontal layer of porous medium saturated by ananofluid is investigated analytically using linear and weakly nonlinear analysis. The model used for the nanofluid incorporates the effect of Brownian motion and thermophoresis. The effect of Raleigh-Darcy number, Lewis number, modified diffusivity ratio, on the stability of the system is investigated. Stationary and Oscillatory modes of convections has been studied. The linear stability analysis is based on normal mode technique, while then on linear theory is based on the truncated representation of Fourier series method. A weekly nonlinear analysis is used to obtain the concentration and thermal Nusselt number. The behavior of the concentration and thermal Nusselt number is investigated by solving the finite amplitude equations. Obtained results have been presented graphically and discussed in details.
\end{abstract}

Keywords: Nanofluid, Porous Medium, Instability, Natural Convection

\section{Introduction}

The term "nanofluid" refers to a liquid containing a suspension of submicronic solid particles (nanoparticles). The term was coined by Choi [1]. The characteristic feature of nanofluids is thermal conductivity enhancement, a phenomenon observed by Masuda et al. [2]. This phenomenon suggests the possibility of using nanofluids in advanced nuclear systems (Buongiorno and $\mathrm{Hu}$ [28]). Nanofluids are mixtures of base fluid such as water or ethylene-glycol with a very small amount of nanoparticles such as metallic or metallic oxide particles $\left(\mathrm{Cu}, \mathrm{Cuo}, \mathrm{Al}_{2} \mathrm{O}_{3}\right)$, having dimensions from 1 to $100 \mathrm{~nm}$. Buongiorno [5] conducted a comprehensive study to account for the unusual behavior of nanofluids based on inertia, Brownian diffusion, thermophoresis, diffusiophoresis, Magnus effects, fluid drainage and gravity settling, and proposed a model incorporating the effect of Brownian diffusion and the thermophoresis. Studies pertaining to thermal conductivity enhancement by nanofluids have been conducted by Eastman et al. [3], Das et al. [4] and others. They claimed a 10-30\% increase in thermal conductivity by using very low concentrations of nanofluid.

Due to application of nanofluid and porous media theory in drying, freezing of foods and applications in every day technology such as microwave heating, rapid heat transfer from computer chips via use of porous metal forms and their use in heat pipes. One of the most significant scientific challenges in the industrial area is cooling, which applies to many diverse productions including microelectronics, transportation and manufacturing. Technological developments such as micro-electronics devices operating at high speeds, high power engines, a brighter optical devices and driving increases thermal loads, requiring advances in cooling.

There are several studies available in which phenomena related to the onset of convectional in a porous medium have been investigated. Few of them are Perlstein [6], Chakrabarti and Gupta [7], Patil and Vidyanathan [8], Vadasz [9]. Convection in porous medium has been studied by many authors including Horton and Roger [10], Lapwood [11], Nield [12], Rudraiah and Malshetty [13], Murray and Chen [14], Bhadauria [15], Vafai [16], Neild and Bejan [17].

Recently, Nield and kuznetsov [18, 19] for the Darcy Model, Nield and Kuznetsov [20] also studied local thermal non-equilibrium and flow past vertical plate for nanofluids. The same problem was studied by Agarwal et al [21] and Bhadauria et al [22]. Umavathi [23] examined the stability of 
porous medium saturated with a nanofluid with thermal conductivity and viscosity dependent on the nanoparticle volume fraction in the case of time dependent wall temperature. A linear and weakly nonlinear stability analysis on the onset of convection in a horizontal porous medium saturated with a Maxwell nanofluid was studied by Umavathi et al [24]. Recently Umavathi and Prathap kumar [25] Analysed the onset of convection in a porous medium layer saturated with an Oldroyd nanofluid. We study the linear and nonlinear analysis of thermal instability in a porous layer saturated by nanofluids in this present article.

\section{Conservation Equations for a Nanofluid}

First, we outline the derivation of conservation equation applicable to a nanofluid in the absence of a solid matrix. Later we modify these equations to the case of a porous medium saturated by the nanofluid. The Buongiorno model treats the nanofluid as a two components mixture (base fluid plus nanoparticles) with the following assumption

$$
\nabla . \mathbf{v}=0
$$

here $\mathbf{v}$ is the nanofluid velocity.

The conservation equation for the nanoparticles in the absence of chemical reactions is

$$
\frac{\partial \phi}{\partial t}+\mathbf{v} \nabla \phi=-\frac{1}{\rho_{p}} \nabla \mathbf{j}_{p}
$$

where $\phi$ is nanoparticle volume fraction, $\rho_{p}$ is the nanoparticle mass density and $\mathbf{j}_{p}$ is the diffusion mass flux for the nanoparticles, given as the sum of two diffusion terms (Brownian diffusion and thermophorosis)by

$$
\mathbf{j}_{p}=\mathbf{j}_{p, B}+\mathbf{j}_{p, T}=-\rho_{p} D_{B} \nabla \phi-\rho_{p} D_{T} \frac{\nabla T}{T}
$$

(Thermophoretic is the "particle" equivalent of the soret effect in gaseous or liquid mixtures) here $D_{B}$ is the Brownian diffusion coefficient given by the Einstein-stokes equation

$$
D_{B}=\frac{k_{B} T}{3 \pi \mu d_{p}}
$$

where $k_{B}$ Boltzmann's constant, $\mu$ is the viscosity of the fluid and $d_{p}$ is the nanoparticle diameter. Use has been made of the expression

$$
\mathbf{V}_{T}=\tilde{\beta} \frac{\mu}{\rho} \frac{\nabla T}{T}
$$

for the thermophoretic velocity $\mathbf{V}_{T}$ here $\rho$ is the fluid density and the proportionality factor $\tilde{\beta}$ is given by

$$
\tilde{\beta}=0.26 \frac{k}{2 k+k_{p}}
$$

where $\mathrm{k}$ and $k_{p}$ are the thermal conductivities of the fluid and the particle material. Hence the thermophoretic diffusion flux is given by

$$
\mathbf{j}_{p, T}=\rho_{p} \phi \mathbf{V}_{T}=-\rho_{p} D_{T} \frac{\nabla T}{T}
$$

where the thermophoretic diffusion coefficient is given by

$$
D_{T}=\tilde{\beta} \frac{\mu}{\rho} \phi
$$

Eq.(2) and (3) then produce the conservation equation in the form

$$
\frac{\partial \phi}{\partial t}+\mathbf{v} . \nabla \phi=\nabla \cdot\left[D_{B} \nabla \phi+D_{T} \frac{\nabla T}{T}\right]
$$

The momentum equation for a nanofluid takes the same form as for a pure fluid, but it should be remembered that $\mu$ is strong function of $\phi$. If one introduce a buoyancy force and adopts the Boussinesq approximation, then the momentum equation can be written as

$$
\rho\left(\frac{\partial \mathbf{v}}{\partial t}+\mathbf{v} \cdot \nabla \mathbf{v}\right)=-\nabla_{p}+\mu \nabla^{2} \mathbf{v}+\rho \mathbf{g},
$$

where

$$
\rho=\phi \rho_{p}+(1-\phi) \rho_{f}
$$

The nanofluid density $\rho$ can be approximated by the base-fluid density $\rho_{f}$ when $\phi$ is small. Then when the Boussinesq approximation is adopted the buoyancy term is approximated by

$$
\rho \mathbf{g} \cong\left[\phi \rho_{p}+(1-\phi)\left\{\rho\left(1-\beta\left(T-T_{0}\right)\right)\right\}\right] \mathbf{g}
$$

The thermal energy equation for a nanofluid can be written as

$$
\rho c\left[\frac{\partial T}{\partial t}+\mathbf{v} \cdot \nabla T\right]=-\nabla \cdot \mathbf{q}+h_{p} \nabla \cdot \mathbf{j}_{p}
$$

where $\mathrm{c}$ is the nanofluid specific heat, $T$ is the nanofluid temperature, $h_{p}$ is the specific enthalpy of the nanoparticle material and $q$ is the energy flux, relative to a frame moving with the nanofluid velocity $\mathbf{V}$, given by

$$
\mathbf{q}=-k \nabla T+h_{p} \mathbf{j}_{p}
$$

where $\mathrm{k}$ is the nanofluid thermal conductivity. Substituting Eq (14) in Eq (13) yields 


$$
\rho c\left[\frac{\partial T}{\partial t}+\mathbf{v} \cdot \nabla T\right]=\nabla \cdot(k \nabla T)-c_{p} \mathbf{j}_{p} \cdot \nabla T
$$

In deriving this equation use has been made of a vector identity and the fact (deriving from assumption (7)) that $\nabla h_{p}=c_{p} \nabla T$, where $c_{p}$ is the nanoparticle specific heat of the material constituting the nanoparticles, while $\mathrm{c}$ is the specific heat (at constant pressure) of the fluid. Then substitution of $\mathrm{Eq}$ (3) in $\mathrm{Eq}$ (15) gives final form

$$
\begin{aligned}
& \rho c\left[\frac{\partial T}{\partial t}+\mathbf{v} . \nabla T\right]=\nabla \cdot(k \nabla T)+ \\
& \rho_{p} c_{p}\left[\begin{array}{c}
D_{B} \nabla \phi . \nabla T+ \\
D_{T} \frac{\nabla T . \nabla T}{T}
\end{array}\right]
\end{aligned}
$$

\section{Conservation Equations for a Porous Medium Saturated by a Nanofluid}

We consider a porous medium whose porosity is denoted by $\varepsilon$ and permeability by $K$. A subscript s will now be used to denote properties of the solid matrix. The Darcy velocity is denoted by $\mathbf{v}_{D}$. This is related to $\mathbf{v}$ by $\mathbf{v}_{D}=\boldsymbol{\varepsilon} \mathbf{v}$. We now have to deal with the following four field equation (corresponding to Eq (1), (10), (16), (9) for total mass, momentum, thermal energy and nanoparticle respectively).

$$
\begin{gathered}
\nabla \cdot \mathbf{v}_{D}=0 \\
\rho\left(\frac{1}{\varepsilon} \frac{\partial \mathbf{v}_{D}}{\partial t}+\frac{1}{\varepsilon^{2}} \mathbf{v}_{D} \cdot \nabla \mathbf{v}_{D}\right)=-\nabla p+ \\
\tilde{\mu} \nabla^{2} \mathbf{v}_{D}-\frac{\mu}{K} \mathbf{v}_{D} \\
+\left[\phi \rho_{p}+(1-\phi)\left\{\rho\left(1-\beta\left(T-T_{0}\right)\right)\right\}\right] \mathbf{g} \\
(\rho c)_{m} \frac{\partial T}{\partial t}+(\rho c)_{f} \mathbf{v}_{D} \nabla T=\nabla\left(k_{m} \nabla T\right)+ \\
\varepsilon(\rho c)_{p}\left[D_{B} \nabla \phi \cdot \nabla T+D_{T} \frac{\nabla T \cdot \nabla T}{T}\right] \\
\frac{\partial \phi}{\partial t}+\frac{1}{\varepsilon} \mathbf{v}_{D} . \nabla \phi=\nabla \cdot\left[D_{B} \nabla \phi+D_{T} \frac{\nabla T}{T}\right]
\end{gathered}
$$

Here we have introduced the effect of viscosity $\tilde{\mu}$, the effective heat capacity $(\rho c)_{m}$ and the effective thermal conductivity $k_{m}$ of the porous medium. In deriving Eq (17)(20) we have assumed that the Brownian motion and thermophoriesis processes remains coherent while volume averages over a representative elementary volume are taken. This assumption can be questioned. In the context of modeling transport in porous media, Eq (17) and (18) are standard. Eq (20) involves just intrinsic quantities in the sense that the average is being taken over the nanofluid only and the solid matrix is not involved. The question thus reduces to whether the terms within the square brackets on the right-hand side of Eq (19) need modification we recall that in nanofluids the particles are so small that for practical purposes they remain in suspension in a uniform manner. We emphasize our assumption that the nanoparticles are suspended in nanofluid using either surfactant or surface charge Technology, something that prevents particles from agglomeration and deposition on the porous matrix. We suggest that then it is reasonable to assume as a first approximation that no modification to Eq (19) is necessary.

\section{Application to the Horton - Rogers - Lapwood Problem}

We select a coordinate frame in which the z-axis is aligned vertically upwards. We consider a horizontal layer of a porous medium confined between the planes $z^{*}=0$ and $z^{*}=H$. From now on asterisks are used to denote dimensional variables (previously an asterisk has not been needed because all the variables were dimensional). Each boundary wall is assumed to be impermeable and perfectly thermally conducting. The temperatures at the lower and upper wall are taken to be $T_{h}^{*}$ and $T_{c}^{*}$, the former being the greater. For simplicity, Darcy's law is assumed to hold and the Oberbeck-Boussinesq approximation is employed. Homogeneity and local thermal equilibrium in the porous medium are assumed The reference temperature is taken to be $T_{c}^{*}$ in the linear theory being applied here the temperature change in the fluid is assumed to be small in comparison with $T_{c}^{*}$ Eq (18)-(20) takes the form

$$
\begin{gathered}
0=-\nabla^{*} p^{*}-\frac{\mu}{K} \mathbf{v}_{D}^{*}+ \\
{\left[\phi^{*} \rho_{p}+\left(1-\phi^{*}\right)\left\{\rho\left(1-\beta\left(T^{*}-T_{C}^{*}\right)\right)\right\}\right] \mathbf{g}} \\
(\rho c)_{m} \frac{\partial T^{*}}{\partial t^{*}}+(\rho c)_{f} \mathbf{v}_{D}^{*} \nabla T=\left(k_{m} \nabla^{* 2} T^{*}\right)+ \\
\varepsilon(\rho c)_{p} \\
{\left[D_{B} \nabla^{*} \phi^{*} \cdot \nabla^{*} T^{*}+\left(D_{T} / T_{C}^{*}\right) \nabla^{*} T^{*} \cdot \nabla^{*} T^{*}\right]} \\
\frac{\partial \phi^{*}}{\partial t^{*}}+\frac{1}{\varepsilon} \mathbf{v}_{D} \cdot \nabla^{*} \varphi^{*}=D_{B} \nabla^{* 2} \varphi^{*}+ \\
\left(\frac{D_{T}}{T^{*}}\right) \nabla^{* 2} T^{*}
\end{gathered}
$$

We write $\mathbf{v}_{D}^{*}=\left(u^{*}, v^{*}, w^{*}\right)$.

We assume that the temperature and the volumetric fraction of the nanoparticles are constant on the boundaries. Thus the boundary conditions are

$$
w^{*}=0, T^{*}=T_{h}^{*}, \phi^{*}=\phi_{0}^{*} \text { at } z^{*}=0
$$




$$
w^{*}=0, T^{*}=T_{C}^{*}, \phi^{*}=\phi_{1}^{*} \text { at } z^{*}=H
$$

We recognize that our choice of boundary conditions imposed on $\phi^{*}$ is somewhat arbitrary. It could be argued that zero particle flux on the boundaries is more realistic physically, but then one is faced with the problem that it appears that no steady-state solution for the basic conduction equations is then possible, so that in order to make analytical progress it is necessary to freeze the basic profile for $\phi^{*}$, and at that stage our choice of boundary conditions is seen to be quite realistic.

We introduce dimensionless variables as follows. We define

$$
\begin{gathered}
(x, y, z)=\left(x^{*}, y^{*}, z^{*}\right) / H, t=t^{*} \alpha_{m} / \sigma H^{2} \\
(u, v, w)=\left(u^{*}, v^{*}, w^{*}\right) H / \alpha_{m}, \quad p=p^{*} K / \mu \alpha_{m} \\
\phi=\frac{\phi^{*}-\phi_{0}^{*}}{\phi_{1}^{*}-\phi_{0}^{*}}, \quad T=\frac{T^{*}-T_{C}^{*}}{T_{h}^{*}-T_{C}^{*}} \\
\text { where } \alpha_{m}=\frac{k_{m}}{\left(\rho c_{p}\right)_{f}}, \quad \sigma=\frac{\left(\rho c_{p}\right)_{m}}{\left(\rho c_{p}\right)_{f}}
\end{gathered}
$$

then $\operatorname{Eq}(17)$ and (21)-(25) take the form

$$
\begin{gathered}
\nabla \cdot \mathbf{v}=0 \\
0=-\nabla p-\mathbf{v}-R m \hat{\mathbf{e}}_{z}+R a_{T} T \hat{\mathbf{e}}_{z}-R n \phi \hat{\mathbf{e}}_{z} \\
\frac{\partial T}{\partial t}+\mathbf{v} . \nabla T=\nabla^{2} T+\frac{N_{B}}{L e} \nabla \phi . \nabla T+ \\
\frac{N_{A} N_{B}}{L e} \nabla T . \nabla T \\
\frac{1}{\sigma} \frac{\partial \phi}{\partial t}+\frac{1}{\varepsilon} \mathbf{v} . \nabla \phi=\frac{1}{L e} \nabla^{2} \phi+\frac{N_{A}}{L e} \nabla^{2} T \\
w=0, T=1, \quad \phi=0 \text { at } z=0 \\
w=0, T=0, \phi=1 \text { at } z=1
\end{gathered}
$$

here $L e=\frac{\alpha_{m}}{D_{B}}, R a_{T}=\frac{\rho g \beta K H \Delta T^{*}}{\mu \alpha_{m}}$,

$$
\begin{gathered}
R m=\frac{\left[\rho_{p} \phi_{0}^{*}+\rho\left(1-\phi_{0}^{*}\right)\right] g K H}{\mu \alpha_{m}}, \\
R n=\frac{\left(\rho_{p}-\rho\right)\left(\varphi_{1}^{*}-\varphi_{0}^{*}\right) g K H}{\mu \alpha_{m}} \\
N_{A}=\frac{D_{T} \Delta T^{*}}{D_{B} T_{c}^{*}\left(\phi_{1}^{*}-\phi_{0}^{*}\right)}, N_{B}=\frac{\varepsilon(\rho c)_{p}}{(\rho c)_{f}}\left(\varphi_{1}^{*}-\varphi_{0}^{*}\right)
\end{gathered}
$$

The parameter $L e$ is a Lewis number and $R a_{T}$ is the familiar thermal Rayleigh-Darcy number. The new parameters $R m$ and $R n$ may be regarded as a basic-density Rayleigh number and a concentration Rayleigh number, respectively. The parameter $N_{A}$ is a modified diffusivity ratio and is somewhat similar to the Soret parameter that arises in cross-diffusion phenomena in solutions, while $N_{B}$ is a modified particle-density increment. In the spirit of the Oberbeck-Boussinesq Eq (29) has been linearize by the neglect of a term proportional to the product of $\phi$ and $T$. This assumption is likely to be valid in the case of small temperature gradients in a dilute suspension of nanoparticles.

\subsection{Basic Solution}

We seek a time-independent quiescent solution of $\mathrm{Eq}(28)$ (33) with temperature and nanoparticle volume fraction varying in the z-direction only, that is a solution of the form

$$
\mathbf{v}=0, \mathrm{~T}=T_{b}(\mathrm{z}), \phi=\phi_{b}(\mathrm{z})
$$

$\mathrm{Eq}(30)$ and (31) reduce to

$$
\begin{gathered}
\frac{d^{2} T_{b}}{d z^{2}}+\frac{N_{B}}{L e} \frac{d \phi_{b}}{d z} \frac{d T_{b}}{d z}+\frac{N_{A} \cdot N_{B}}{L e}\left(\frac{d T_{b}}{d z}\right)^{2}=0 \\
\frac{d^{2} \phi_{b}}{d z^{2}}+N_{A} \frac{d^{2} T_{b}}{d z}=0
\end{gathered}
$$

Using the boundary conditions (32) and (33) Eq (41) may be integrated to give

$$
\phi_{b}=-N_{A} T_{b}+\left(1-N_{A}\right) Z+N_{A}
$$

And substitution of this into Eq (34) gives

$$
\frac{d^{2} T_{b}}{d Z^{2}}+\frac{\left(1-N_{A}\right) N_{B}}{L e} \frac{d T_{b}}{d Z}=0
$$

The solution of Eq (37) satisfying Eq (32) and (33) is

$$
T_{b}=\frac{1-e^{-\left(1-N_{A}\right) N_{B}(1-Z) / L e}}{1-e^{-\left(1-N_{A}\right) N_{B} / L e}}
$$

The remainder of the basic solution is easily obtained by first substituting in Eq (42) to obtain $\phi_{b}$ and then using integration of Eq (29) to obtain $P_{b}$.

According to Buongiorno [5], for most nanofluids investigated so far $L e /\left(\phi_{1}^{*}-\phi_{0}^{*}\right)$ is large, of order $10^{5}-10^{6}$, and since the nanoparticle fraction decrement is typically no smaller than $10^{3}$ this means so that Le is large, of order $10^{2}-$ $10^{3}$, while $N_{A}$ is no greater than about 10 . Then the exponents in Eq (37) and (38) are small and so to a good approximation one has

$$
T_{b}=1-z
$$

and so 


$$
\varphi_{b}=z
$$

\subsection{Perturbation Solution}

We now superimpose perturbations on the basic solution. We write

$$
\mathbf{v}=\mathbf{v}^{\prime}, p=p_{b}+p^{\prime}, T=T_{b}+T^{\prime}, \varphi=\varphi_{b}+\varphi^{\prime}
$$

Substitute in Eq (28)-(33), and linearize by neglecting products of primed quantities. The following equations are obtained when Eq (39) and (40) are used.

$$
\begin{gathered}
\nabla \cdot \mathbf{v}^{\prime}=0 \\
0=-\nabla p^{\prime}-\mathbf{v}^{\prime}+R a_{T} T^{\prime} \hat{\mathbf{e}}_{z}-R n \phi^{\prime} \hat{\mathbf{e}}_{z} \\
\frac{\partial T^{\prime}}{\partial t}-w^{\prime}=\nabla^{2} T^{\prime}+\frac{N_{B}}{L e}\left(\frac{\partial T^{\prime}}{\partial z}-\frac{\partial \varphi^{\prime}}{\partial z}\right) \\
-\frac{2 N_{A} N_{B}}{L e} \frac{\partial T^{\prime}}{\partial z} \\
\frac{1}{\sigma} \frac{\partial \varphi^{\prime}}{\partial t}+\frac{1}{\varepsilon} w^{\prime}=\frac{1}{L e} \nabla^{2} \varphi^{\prime}+\frac{N_{A}}{L e} \nabla^{2} T^{\prime} \\
w^{\prime}=0, T^{\prime}=0, \varphi^{\prime}=0 \text { at } z=0 \text { and at } z=1
\end{gathered}
$$

It will be noted that the parameter $R m$ is not involved in these and subsequent equations. It is just a measure of the basic static pressure gradient.

For the case of a regular fluid (not a nanofluid) the parameters $R n, N_{A}$ and $N_{B}$ are zero, the second term in Eq (45) is absent because $d \phi_{b} / d z=0$ and then Eq (45) is satisfied trivially. The remaining equations are reduced to the familiar equations for the Horton-Roger-Lapwood problem.

The six unknowns $u^{\prime}, v^{\prime}, w^{\prime}, p^{\prime}, T^{\prime}, \varphi^{\prime}$ can be reduced to three by operating on Eq (43) with $\hat{e}_{z}$ curl curl and using the identity curl curl $\equiv \operatorname{grad} \operatorname{div}-\nabla^{2}$ together with Eq (42).

The result is

$$
\nabla^{2} w^{\prime}=R a_{T} \nabla_{H}^{2} T^{\prime}-R n \nabla_{H}^{2} \phi^{\prime}
$$

here $\nabla_{H}^{2}$ is the two-dimensional Laplacian operator on the horizontal plane.

The differential Eq (47), (44), (45) and the boundary conditions (46) constitute a linear boundary-value problem that can be solved using the method of normal modes.

We write

$$
\left(w^{\prime}, T^{\prime}, \phi^{\prime}\right)=[W(z), \theta(z), \Phi(z)] \exp (s t+i l x+i m y)
$$

and substitute into the differential equations to obtain

$$
\left(D^{2}-\alpha^{2}\right) W+R a_{T} \alpha^{2} \Theta-R n \alpha^{2} \Phi=0
$$

$$
\begin{aligned}
W+\left(D^{2}+\right. & \left.\frac{N_{A}}{L e} D-\frac{2 N_{A} \cdot N_{B}}{L e} D-\alpha^{2}-s\right) \Theta \\
-\frac{N_{B}}{L e} D \Phi= & 0 \\
& \frac{1}{\varepsilon} W-\frac{N_{A}}{L e}\left(D^{2}-\alpha^{2}\right) \Theta- \\
& \left(\frac{1}{L e}\left(D^{2}-\alpha^{2}\right)-\frac{s}{\sigma}\right) \Phi=0
\end{aligned}
$$$$
W=0, \Theta=0 \text {, at } Z=0 \text { and at } Z=1
$$

Where

$$
D \equiv \frac{d}{d z} \text { and } \alpha^{2}=\left(l^{2}+m^{2}\right)^{1 / 2}
$$

thus $\alpha$ is a dimensionless horizontal wave number.

For neutral stability the real part of $\mathrm{s}$ is zero. Hence we now write $s=i \omega$, where $\omega$ is real and is a dimensionless frequency. We now employ a Galerkin-type weighted residuals method to obtain an approximate solution to the system of Eq(49)-(52). We choose as trial functions (satisfying the boundary conditions)

$$
W_{p}=\Theta_{p}=\Phi=\sin p \pi z ; p=1,2,3 \ldots \ldots
$$

write

$$
\mathrm{W}=\sum_{p=1}^{N} A_{p} W_{p}, \Theta=\sum_{p=1}^{N} B_{p} \Theta_{P}, \Phi=\sum_{p=1}^{N} C_{p} \Theta_{p}
$$

substitute into Eq (49)-(51), and make the expressions on the lefthand sides of those equations (the residuals) orthogonal to the trial functions, there by obtaining a system of $3 \mathrm{~N}$ linear algebraic equations in the $3 \mathrm{~N}$ unknowns $\mathrm{Ap}, \mathrm{Bp}, \mathrm{Cp}, \mathrm{p}=1$, $2, \ldots . \mathrm{N}$. The vanishing of the determinant of coefficients produces the eigenvalue equation for the system. One can regard $R a_{T}$ as the eigenvalue. Thus $R a_{T}$ is found in terms of the other parameters

\section{Linear Stability Analysis}

\subsection{Non-oscillatory Convection}

First, we consider the case of non-oscillatory instability, when $\omega=0$ for a first approximation we take $\mathrm{N}=1$.This produces the result

$$
R a_{T}=\frac{\left(\pi^{2}+\alpha^{2}\right)^{2}}{\alpha^{2}}-\left(N_{A}+\frac{L e}{\varepsilon}\right) R n .
$$

Finding the minimum as a varies results in

$$
R a_{T}=4 \pi^{2}-\left(N_{A}+\frac{L e}{\varepsilon}\right) R n .
$$

with the minimum being attained at $\alpha=\pi$. We recognize that in the absence of nanoparticles we recover the well-known 
result that the critical Rayleigh number is equal to $4 \pi^{2}$. Usually when one employs a single-term Galerkin approximation in this context one gets an overestimate by about 3\% (e.g. 1750 instead of 1708 in the case of the standard Bénard problem) but in this case the approximation happens to give the exact result. As we have noted, for a typical nanofluid Le is of order $10^{2}-10^{3}$ and $N_{A}$ is not much greater than 10 . Hence the coefficient of $R n$ in Eq (57) is large and negative. Thus under the approximations we have made so far we have the result that the presence of nanoparticles lowers the value of the critical Rayleigh number, usually by a substantial amount, in the case when $R n$ is positive, that is when the basic nanoparticle distribution is a top-heavy one. It will be noted that in Eq (57) the parameter $N_{B}$ does not appear. The instability is almost purely a phenomenon due to buoyancy coupled with the conservation of nanoparticles. It is independent of the contributions of Brownian motion and thermophoresis to the thermal energy equation. Rather, the Brownian motion and thermophoresis enter to produce their effects directly into the equation expressing the conservation of nanoparticles so that the temperature and the particle density are coupled in a particular way and that results in the thermal and concentration buoyancy effects being coupled in the same way. It is useful to emphasize this by rewriting $\mathrm{Eq}$ (57) in the form

$$
R a_{T}+\left(N_{A}+\frac{L e}{\varepsilon}\right) R n=4 \pi^{2}
$$

and noting that the left-hand side is the linear combination of the thermal Rayleigh number $R a$ and the concentration Rayleigh number $R n$. The problem is analogous to the double- diffusive problem discussed in Section 9.1.1 of Nield and Bejan [26]. It is also analogous to the bioconvection problem discussed by Kuznetsov and Avramenko [27]. We have defined $R n$ in a way so that it is positive when the applied particle density increases upwards (the destabilizing situation). We note that $R a_{T}$ takes a negative value when $R n$ is sufficiently large. In this case the destabilizing effect of concentration is so great that the bottom of the fluid layer must be cooled relative to the top in order to produce a state of neutral stability. We emphasize that the simple expression in $\mathrm{Eq} \mathrm{(57)} \mathrm{arises} \mathrm{because} \mathrm{the} \mathrm{Lewis} \mathrm{number} \mathrm{has} \mathrm{been}$ assumed to be large. In order to estimate the contribution of the terms involving $N_{B}$ we have investigated the two-term Galerkin results. The expression in the eigenvalue equation is complicated and it is difficult to make a statement that is simultaneously precise, simple and general. However, it is clear that the functions of $N_{B}$ are of second degree. We conclude that for practical purposes Eq (58) is a good approximation.

\subsection{Oscillatory Convection}

We now consider the case $\omega \neq 0$. We confine ourselves to the one-term Galerkin approximation. The eigen value equation now takes the form

$$
\begin{aligned}
& R a_{T} \alpha^{2}\left(\frac{J}{L e}+\frac{i \omega}{\sigma}\right)+R n \alpha^{2}\left(\frac{N A}{L e}+\frac{(J+i \omega)}{\varepsilon}\right) \\
& =J(J+i \omega)\left(\frac{J}{L e}+\frac{i \omega}{\sigma}\right)
\end{aligned}
$$

Where for shorthand we have written

$$
J=\pi^{2}+\alpha^{2}
$$

\section{Non-linear Stability Analysis}

For simplicity, we consider the case of two dimensional rolls, assuming all physical quantities to be independent of $y$. Eliminating the pressure and introducing the stream function we obtain

$$
\begin{array}{r}
\nabla^{2} \Psi+R a_{T} \frac{\partial T}{\partial x}-R n \frac{\partial S}{\partial x}=0 \\
\frac{\partial T}{\partial t}+\frac{\partial \Psi}{\partial x}=\nabla^{2} T+\frac{\partial(\Psi, T)}{\partial(x, z)} \\
\frac{1}{\sigma} \frac{\partial S}{\partial t}+\frac{1}{\varepsilon} \frac{\partial \Psi}{\partial x}=\frac{1}{L e} \nabla^{2} S+\frac{N_{A}}{L e} \nabla^{2} T \\
+\frac{1}{\varepsilon} \frac{\partial(\Psi, S)}{\partial(x, z)}
\end{array}
$$

We solve Eq1-3 subjecting them to stressfree, isothermal,iso-nano concentration boundary conditions

$$
\psi=\frac{\partial^{2} \psi}{\partial z^{2}}=T=\phi=0 \text { at } z=0,1
$$

To per forma local non-linear stability analysis, we take the following Fourier expressions

$$
\begin{aligned}
& \psi=\sum_{n=1}^{\infty} \sum_{m=1}^{\infty} A_{m n}(t) \operatorname{Sin}(\max ) \sin (n \pi z) \\
& T=\sum_{n=1}^{\infty} \sum_{m=1}^{\infty} B_{m n}(t) \cos (\max ) \sin (n \pi z) \\
& \phi=\sum_{n=1}^{\infty} \sum_{m=1}^{\infty} C_{m n}(t) \cos (\max ) \sin (n \pi z)
\end{aligned}
$$

Further, we take the modes $(1,1)$ for stream function, and $(0,2)$ and $(1,1)$ for temperature, and nanoparticle concentration, to get

$$
\begin{gathered}
\psi=A_{1}(t) \operatorname{Sin}(\mathrm{ax}) \sin (\pi z) \\
T=A_{2}(t) \cos (\mathrm{ax}) \sin (\pi z)+A_{3}(t) \sin (2 \pi z) \\
S=A_{4}(t) \cos (\mathrm{ax}) \sin (\pi z)+A_{5}(t) \sin (2 \pi z)
\end{gathered}
$$

Where the amplitudes $A_{1}(t), A_{2}(t), A_{3}(t), A_{4}(t), A_{5}(t)$ are function of time and are to be determined. Taking the 
orthogonality condition with the eigen functions associated with the considered minimal model, we get

$$
\begin{gathered}
A_{1}(t)=\frac{a}{\delta^{2}}\left[R n A_{4}(t)-R a_{T} A_{2}(t)\right] \\
\frac{d A_{2}(t)}{d t}=-\left[a A_{1}(t)+\delta^{2} A_{2}(t)+a \pi A_{1}(t) A_{3}(t)\right] \\
\frac{d A_{3}(t)}{d t}=-4 \pi^{2} A_{3}(t)+\frac{a \pi}{2} A_{1}(t) A_{2}(t) \\
\frac{d A_{4}(t)}{d t}=-\frac{1}{\sigma}\left[\begin{array}{l}
\frac{1}{\varepsilon} a A_{1}(t)+ \\
\delta^{2}\left[\frac{A_{4}(t)}{L e}+\frac{N_{A}}{L e} A_{2}(t)\right] \\
+\frac{1}{\varepsilon} a A_{1}(t) A_{5}(t)
\end{array}\right] \\
\frac{d A_{5}(t)}{d t}=-\frac{1}{\sigma}\left[\begin{array}{l}
\frac{1}{L e} \\
-\frac{1}{\varepsilon} \frac{a \pi}{2} \pi^{2} A_{5}(t)+4 \pi^{2} A_{3}(t) \frac{N_{A}}{L e}
\end{array}\right]
\end{gathered}
$$

thus we get

$$
\begin{gathered}
A_{1}(t)=\frac{a}{\delta^{2}}\left[R n A_{4}(t)-R a_{T} A_{2}(t)\right] \\
D_{2}=-\left[a A_{1}(t)+\delta^{2} A_{2}(t)+a \pi A_{1}(t) A_{3}(t)\right] \\
D_{3}=-4 \pi^{2} A_{3}(t)+\frac{a \pi}{2} A_{1}(t) A_{2}(t) \\
D_{4}=-\frac{1}{\sigma}\left[\frac{1}{\varepsilon} a A_{1}(t)+\delta^{2}\left[\frac{A_{4}(t)}{L e}+\frac{N_{A}}{L e} A_{2}(t)\right]\right. \\
\left.+\frac{1}{\varepsilon} a A_{1}(t) A_{5}(t)\right] \\
D_{5}=-\frac{1}{\sigma}\left[\frac{1}{L e} 4 \pi^{2} A_{5}(t)+4 \pi^{2} A_{3}(t) \frac{N_{A}}{L e}\right. \\
\left.-\frac{1}{\varepsilon} \frac{a \pi}{2} A_{1}(t) A_{4}(t)\right] \\
\text { and } D_{2}=D_{3}=D_{4}=D_{5}=0
\end{gathered}
$$

One may also conclude that the trajectories of the above equations will be confined to the finiteness of the ellipsoid. Thus, the effect of the parameters $R n, L e, N_{A}$ on the trajectories is to attract them to a set of measure zero, or to a fixed point to say

\subsection{Heat and Nanoparticle Concentration Transport}

The thermal Nusselt number, $N u_{f}(t)$ is defined as

$$
\begin{aligned}
& N u_{f}(t)= \\
& \frac{\text { Heat transport by (conduction }+ \text { convection })}{\text { Heat transport by conduction }}
\end{aligned}
$$

$$
=1+\left[\frac{\int_{0}^{2 \pi / a}\left(\frac{\partial T}{\partial Z}\right) d x}{\int_{0}^{2 \pi / a}\left(\frac{\partial T_{B}}{\partial z}\right) d x}\right]_{z=0}
$$

Substituting expressions (45) and (73) in Eq (83) we get

$$
N u_{f}(t)=1-2 \pi A_{3}(t)
$$

The nanoparticle concentration Nusselt number, $N u_{\varphi}(t)$ is defined similar to the thermal Nusselt number. Following the procedure adopted for arriving at $N u(t)$, one can obtain the expression for $N u_{\varphi}(t)$ in the form

$$
N u_{\phi}(t)=\left(1-2 \pi A_{5}(t)\right)+N_{A}\left(1-2 \pi A_{3}(t)\right)
$$

\section{Results and Discussions}

The expressions of thermal Rayleigh number for stationary and oscillatory convections are given by equations (56) and (59) respectively
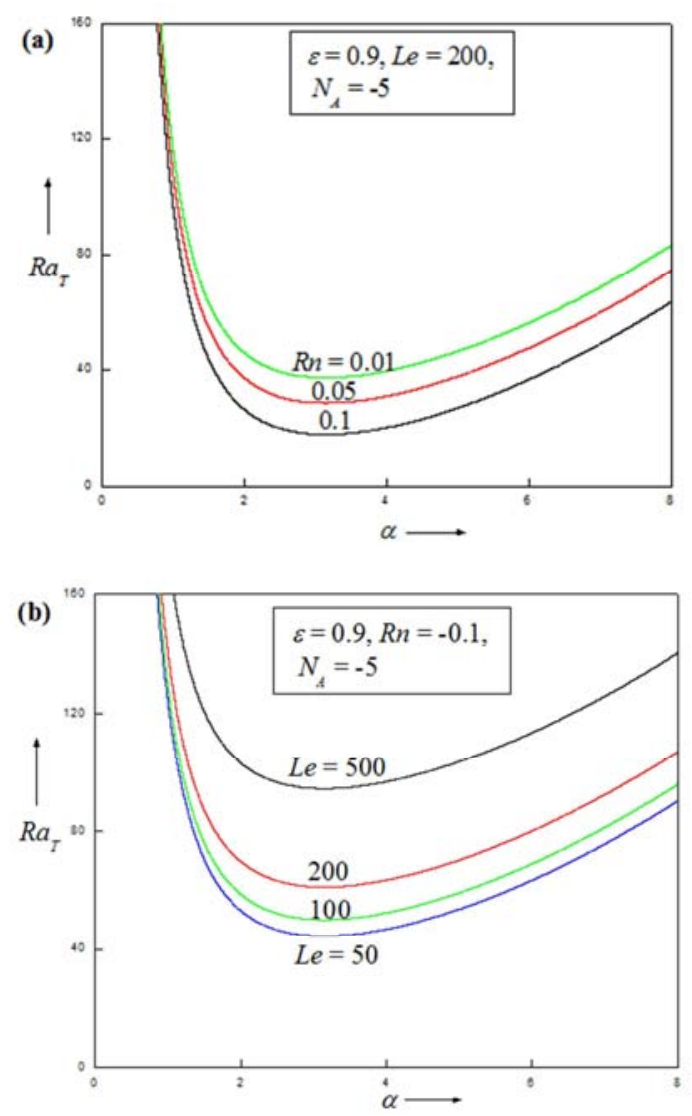

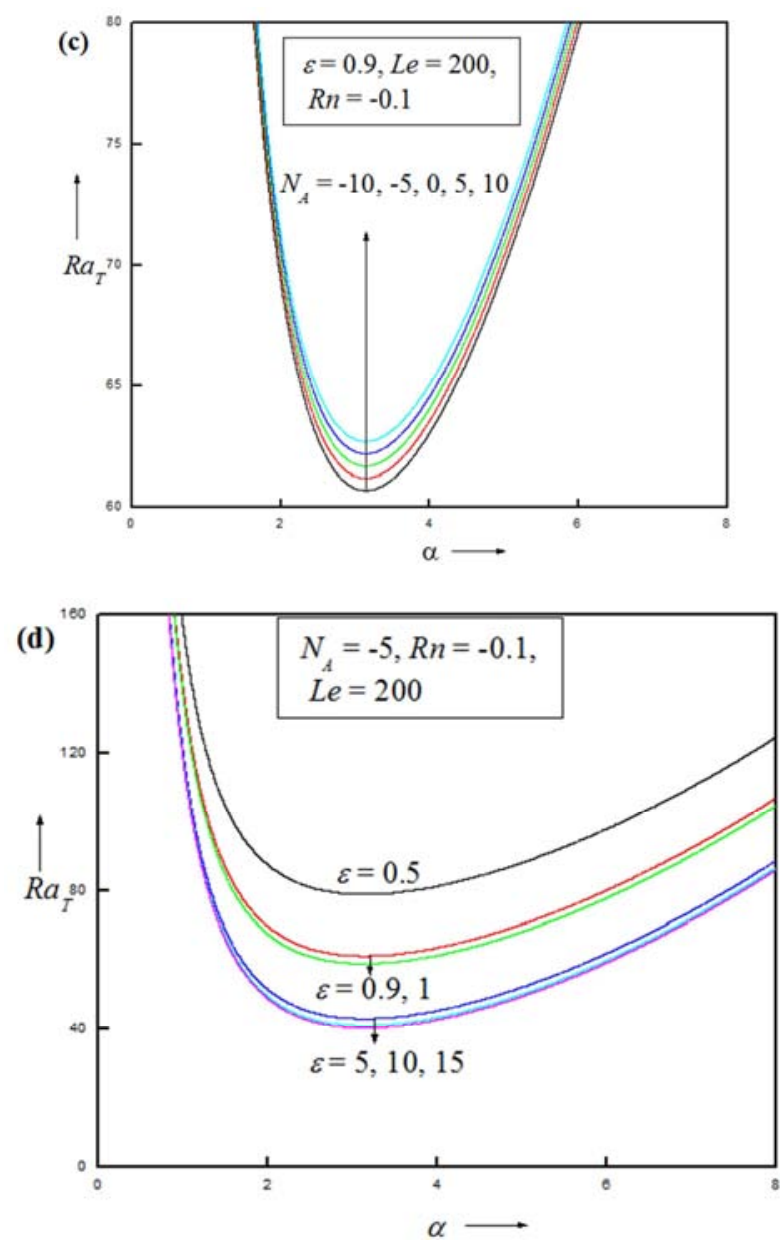

Figure 1. Neutral curves on stationary convection for different values of, (a)nanoparticle concentration Rayleigh number Rn (b)Lewis number Le (c)modified diffusivity ratio $N_{A}$ (d)porsity $\mathcal{E}$.

Figure 1a-d shows the effect of various parameters on the neutral stability curves for stationary convection for $R n=$ $0.1, L e=200, N_{A}=-5, \varepsilon=0.9$, with variation in one of the parameters. The effect of nanoparticle concentration Rayleigh number $R n$ is shown in Figure 1a. It is shown that the thermal Rayleigh number decreases with increase in nanoparticle concentration Rayleigh number $R n$, which means that nanoparticle concentration Rayleigh number $R n$ destabilizes the system. It should be noted that the negative value of $R n$ indicates a bottom-heavy case, while a positive value indicates a top-heavy case. The effect of Lewis number $L e$ on the thermal Rayleigh number is shown in Figure.1b. One can see that the thermal Rayleigh number increases with increase in Lewis number, indicating that the Lewis number stabilizes the system. The effect of modified diffusivity ratio $N_{A}$ on the thermal Rayleigh number is shown in Figure 1c, it is shown in Figure. $1 \mathrm{c}$ that as $N_{A}$ increases $R a_{T}$ increase and hence $N_{A}$ has a stabilizing effect on the system. From Figure $1 \mathrm{~d}$, one can observe that as porosity $\varepsilon$ increases, thermal Rayleigh number decreases which means that the porosity advances the onset of convection.
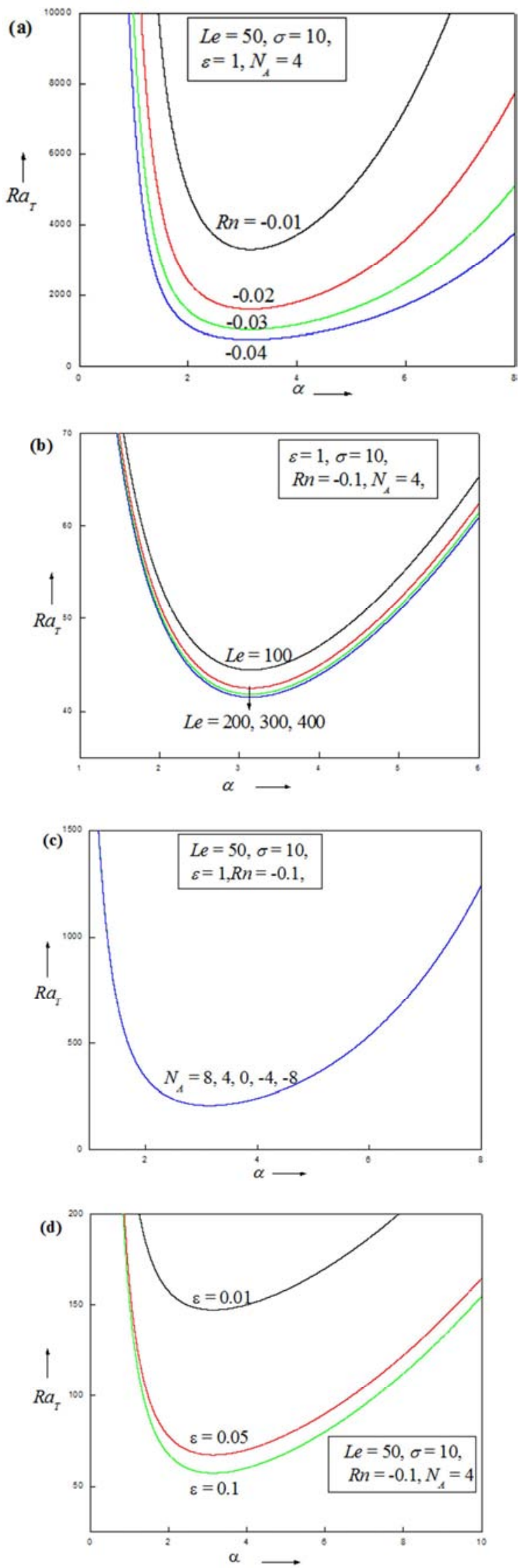


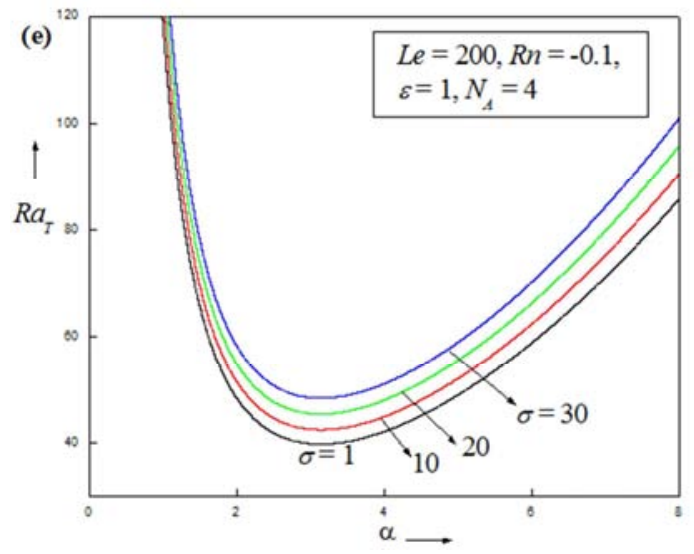

Figure 2. Neutral curves on oscillatory convection for different values of (a) nanoparticle concentration Rayleigh number Rn (b) Lewis number Le (c) modified diffusivity ratio $N_{A}(d)$ porosity $\mathcal{E}$ (e) Thermal Capacity $\sigma$.

Figure 2a-e displays the variation of thermal Rayleigh number for oscillatory convection with respect to various parameters. In Figure 2a it is seen that for negative values of $R n$ (bottom heavy case). The thermal Rayleigh number decreases as $R n$ increases which will delay the onset of convection. As the Lewis number Le increases the thermal Rayleigh number $R a_{T}$ decreases as seen in Figure $2 \mathrm{~b}$ which imply that Lewis number $L e$ destabilizes the system. The modified diffusivity ratio $N_{A}$ do not show any effect on the oscillatory convection (Figure 2c). From the picture 2d, one can reveal that the porosity $\mathcal{E}$ destabilizes the system for oscillatory convection, that is an increase in $\varepsilon$ decreases the thermal Rayleigh number. As the thermal capacity ratio $\sigma$ increases, the thermal Rayleigh number also increases as can be observed in Figure 2e, which implies that $\sigma$ has a stabilizing effect on the system for oscillatory convection.

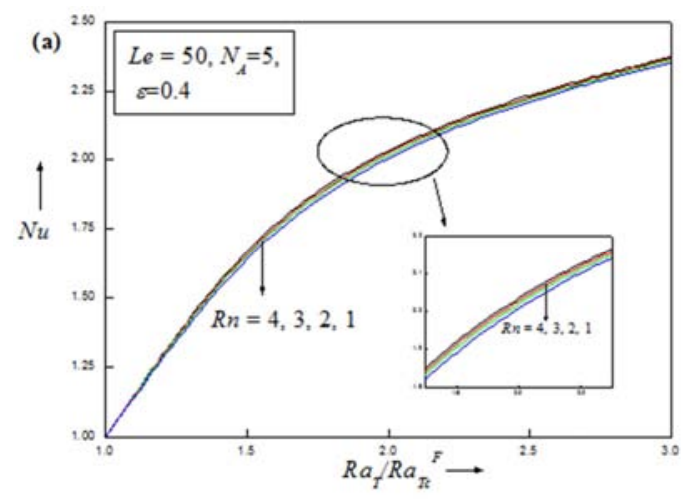

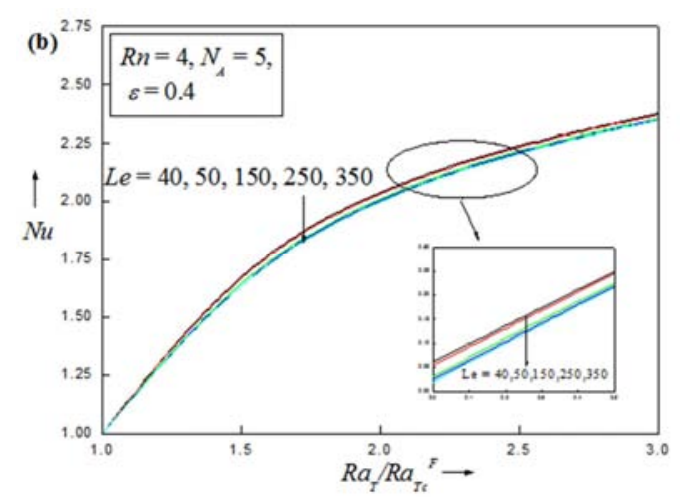

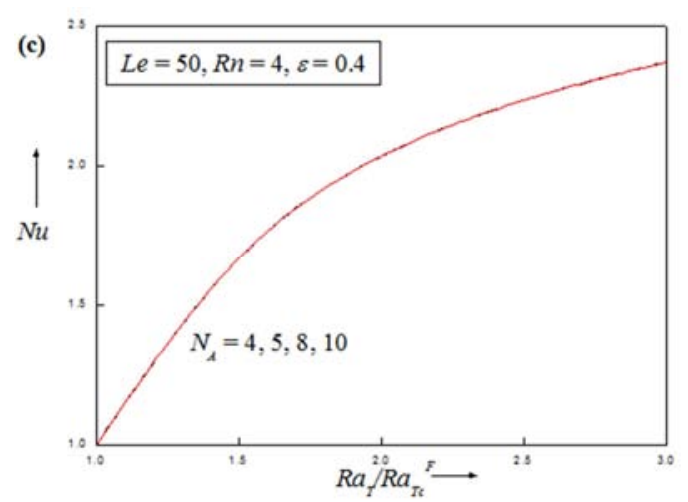

Figure 3. Variation of Nusselt number Nu with critical Rayleigh Number for different values of (a) Nanoparticle concentration Rayleigh number Rn,(b) Lewis number Le,(c) Modified diffusivity ratio $N_{A}$.

The nonlinear analysis provides not only the onset threshold of finite amplitude motions but also the information of heat and mass transports in terms of Nusselt $N u$ and Sherwood $S h$ numbers. The $N u$ and $S h$ are computed as the functions of $R a_{T}$ (thermal Rayleigh number), and the variations of these non-dimensional numbers with $R a_{T}$ for different parameter values are depicted in Figures $3 \mathrm{a}-\mathrm{c}$ and $4 a-c$ respectively. In Figures 3a-c and 4a-c it is observed that in each case, Sherwood number is always greater than Nusselt number and both Nusselt number and Sherwood number start with the conduction state value 1 at the point of onset of steady finite amplitude convection. When $R a_{T}$ is increased beyond $R a_{T c}$, there is a sharp increase in the values of both $N u$ and $S h$. However further increase in Ra will not change $N u$ and $S h$ significantly. It is to be noted that the upper bound of $\mathrm{Nu}$ is 3 (similar results were obtained by Malashetty et al.). It should also be noted that the upper bound of Sh is not 3 (similar results were obtained by Bhadauria et al.). The upper bound of $N u$ remains 3 only for both clear and nanofluid. Whereas, the upper bound for $S h$ for clear fluid is 3 but for nanofluid it is not fixed. 

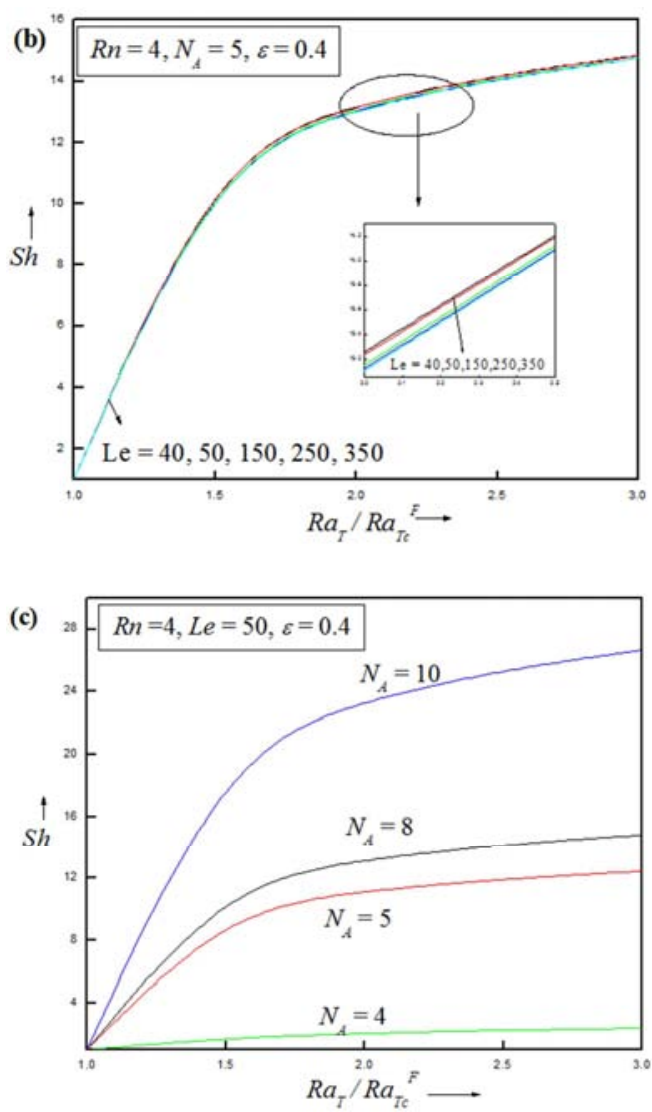

Figure 4. Variation of Sherwood number Sh with critical Rayleigh Number for different values of (a) Nanoparticle concentration Rayleigh number Rn, (b) Lewis number Le,(c) Modified diffusivity ratio $N_{A}$.

In Figures. 3a and $4 \mathrm{a}$ we observe that as the concentration Rayleigh number $R n$ increases, the value of $N u$ and $S h$ decreases, thus showing a decrease in the rate of heat and mass transport. Figures. $3 \mathrm{~b}$ and $4 \mathrm{~b}$ shows that as Lewis number increases both $\mathrm{Nu}$ and $S h$ decreases, which imply that increasing the Lewis number suppresses the heat and mass transport. In Figures. $3 \mathrm{c}$ and $4 \mathrm{c}$ we observe that on increasing modified diffusivity ratio $N_{A}$, there is no effect on the Nusselt number whereas it increases the Sherwood number (which is similar result observed by Bhadauria et al [29]).

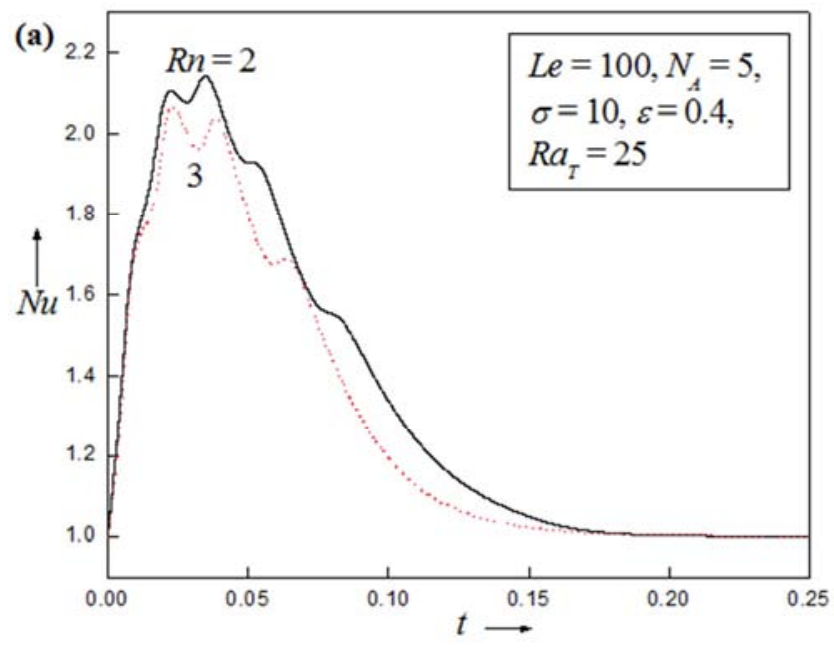

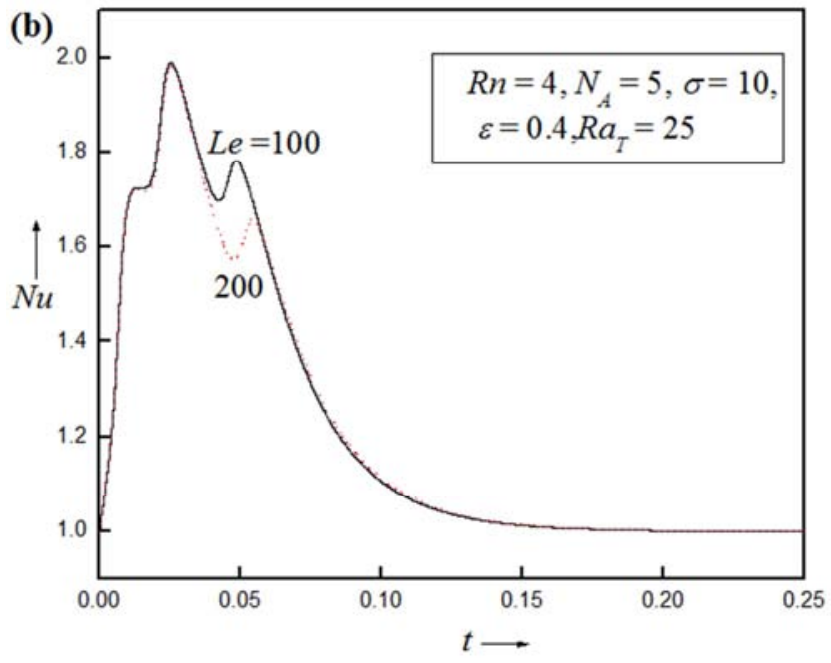

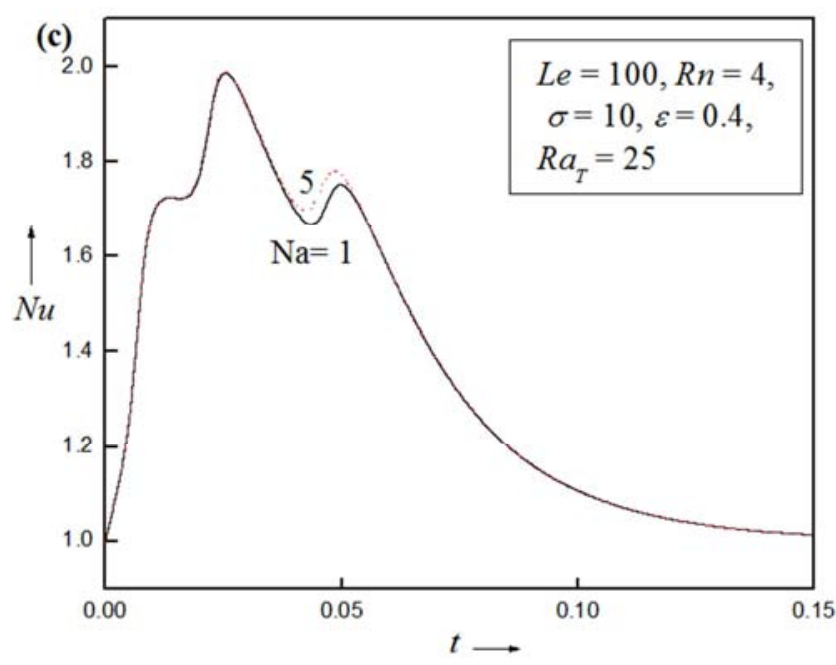

Figure 5. Variation of Nusselt number Nu with Rayleigh Number for different values of, (a) Nanoparticle concentration Rayleigh number Rn, (b) Lewis number Le, (c) Modified diffusivity ratio $N_{A}$

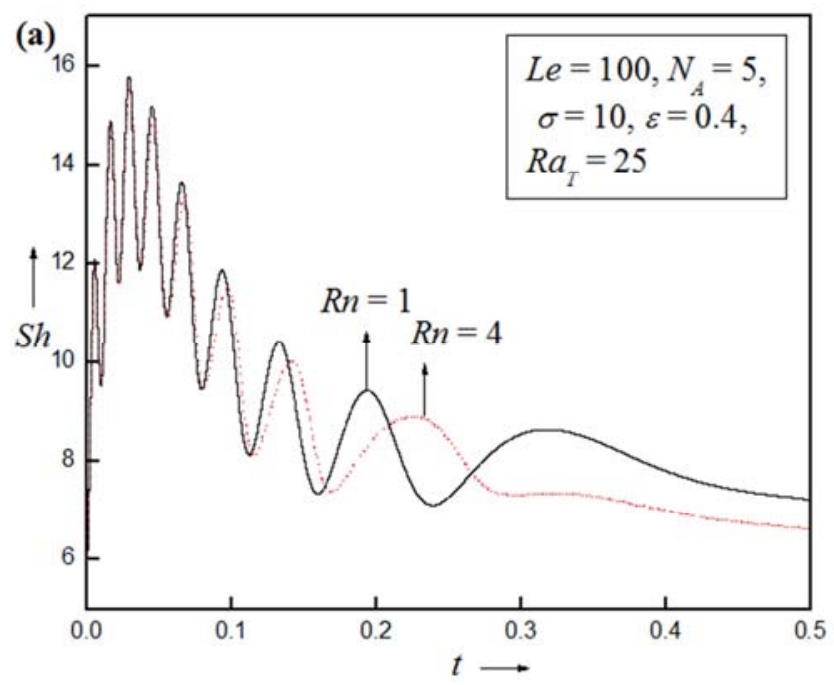



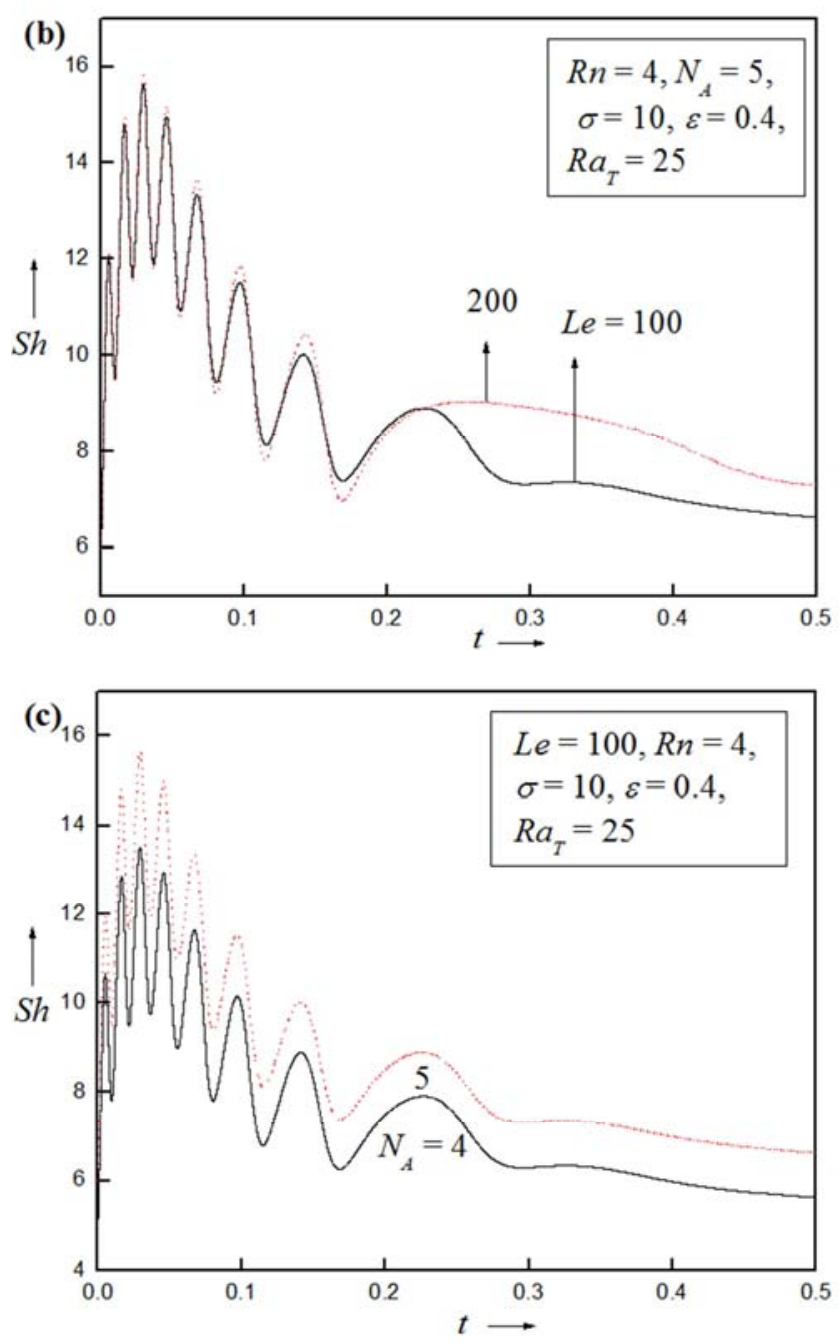

Figure 6. Variation of Sherwood number Sh with Rayleigh Number for different values of (a) Nanoparticle concentration Rayleigh number Rn, (b)Lewis number Le,(c) Modified diffusivity ratio $N_{A}$.

In Figure 5a it is observed that as Rayleigh number $R n$ increases Nusselt number $N u$ decrease, thus showing a decrease in the heat transport. In Figure $5 b$ as Lewis number $\mathrm{Le}$ increases the Nusselt number $\mathrm{Nu}$ decreases indicating there is retardation on heat transport. The modified diffusivity ratio $N_{A}$ enhances the heat transport as see in Figure 5c. In Figure $6 \mathrm{a}$ as nanoparticle concentration Rayleigh number $R n$ increases the Sherwood number decreases, which implies the suppress of mass transport. In Figures $6 \mathrm{~b}$ and $6 \mathrm{c}$ the mass transport is enhanced for Lewis number $L e$ and modified diffusivity ratio $N_{A}$.

\section{Conclusions}

We considered linear stability analysis in a horizontal porous medium saturated by a nanofluid, heated from below and cooled from above, using Darcy model which incorporate the effect of Brownian motion along with thermophoresis. Further the viscosity and conductivity dependence on nanoparticle fraction was also adopted following Tiwari and Das [30]. Linear analys is has been made using normal mode technique. However for weakly nonlinear analysis truncated Fourier series representation having only two terms is considered. We draw the following conclusions.

For stationary convection Lewis number Le, modified diffusivity ratio $N_{A}$, has a stabilizing effect while nanoparticle concentration Rayleigh number $R n$ and porosity destabilize the system. For oscillatory convection thermal capacity ratio $\sigma$, stabilizes the system where as nanoparticle concentration Rayleigh number $R n$, Lewis number $L e$ and porosity destabilizes the system. For steady finite amplitude motions, the heat and mass transported creases within crease in the values of nanoparticle concentration Rayleigh number $R n$, Lewis number $L e$. The mass transport increases with increase in modified diffusivity ratio.

The transient Nusselt number and Sherwood number increases with increase in Lewis number $L e$ and modified diffusivity ratio $N_{A}$ and decreases with nanoparticle concentration Rayleigh number $R n$.The effect of time on transient Nusselt number and Sherwood number is found to be oscillatory when $t$ is small. However, when $t$ becomes very large both the transient Nusselt and Sherwood value approaches to the steady value.

\section{Nomenclature}

$c$ Nanofluid sp ecific heat at constant pressure

$c_{p}$ Specific heat of the nanoparticle material

$(\rho c)_{m}$ Effective heat capacity of the porous material

$d_{p}$ Nanopartical diameter

$D_{B}$ Brownian diffusion coefficient $\left(\mathrm{m}^{2} / \mathrm{s}\right)$, given by Eq.(4)

$D_{T}$ Thermophoretic diffusion coefficient $\left(\mathrm{m}^{2} / \mathrm{s}\right)$, given by Eq.(8)

$h_{p}$ Specific enthalpy of the nanopartical material

$H$ Dimensional layer depth $(m)$

$\mathbf{j}_{p}$ Diffusion mass flux for the nanoparticales, given by Eq.(7)

$\mathbf{j}_{p, T}$ Thermophoretic diffusion, given by Eq.(7)

$k$ Thermal conductivity of the nanofluid (W/m K)

$k_{B}$ Boltzmann's constant

$k_{m}$ Overall thermal conductivity of the porous medium saturated by the nanofluid

$k_{p}$ Thermal conductivity of the particle material

K Permeability $\left(\mathrm{m}^{2}\right)$ Le Lewis number

$N_{A}$ Modified diffusivity ratio

$N_{B}$ Modified particle density increment

$p^{*}$ Pressure $(\mathrm{Pa})$

$p$ Dimensionless pressure, $p^{*} K / \mu \alpha_{f}$

q Energy flux relative to a frame moving with the nanofluid velocity $\mathbf{v}$ 
$R a_{T}$ Thermal Rayleigh- Darcy number

$R m$ Basic-density Rayleigh number

$R n$ Concentration Rayleigh number

$t^{*}$ Time (s)

$t$ Dimensionless time, $t^{*} \alpha_{f} / H^{2}$

$T^{*}$ Nanofluid temperature (K)

$T$ Dimensionless temperature, $\frac{T^{*}-T_{c}^{*}}{T_{h}^{*}-T_{c}^{*}}$

$T_{c}^{*}$ Temperature at the upper wall $(\mathrm{K})$

$T_{h}^{*}$ Temperature at the lower wall $(\mathrm{K})$

v Nanofluid velocity $(\mathrm{m} / \mathrm{s})$

$\mathbf{v}_{D}$ Darcy velocity $\boldsymbol{\varepsilon} \mathbf{v}$

$\mathbf{v}_{D}^{*}$ Dimentional Darcy velocity

$\mathbf{V}_{T}$ Themophoreatic velocity

$(u, v, w)$ Dimensionless Darcy velocity components $\left(u^{*}, v^{*}, w^{*}\right) H / \alpha_{m}(\mathrm{~m} / \mathrm{s})$

$(x, y, z)$ Dimensionless Cartesian coordinate $\left(x^{*}, y^{*}, z^{*}\right) / H ; z$ is the vertically upward coordinate

$\left(x^{*}, y^{*}, z^{*}\right)$ Cartesian coordinates

\section{Greek Symbols}

$\alpha_{m}$ Thermal diffusivity of the porous medium

$\tilde{\beta}$ Proportionality factor, given by Eq.(6)

$\mu$ Viscosity of the fluid

$\tilde{\mu}$ Effective viscosity of the porous medium

$\rho$ Fluid density

$\rho_{p}$ Nanoparticle mass density

$\sigma$ Thermal capacity ratio

$\phi^{*}$ Nanoparticle volume fraction

$\phi$ Relative nanoparticle volume fraction, $\frac{\phi^{*}-\phi_{0}^{*}}{\phi_{1}^{*}-\phi_{0}^{*}}$

\section{Superscripts}

*Dimensional variable

'Perturbed variable

\section{Subscripts}

b Basic Solution

f Fluid

p Particle

\section{References}

[1] Choi, S.: Enhancing thermal conductivity of fluids with nanoparticles. In: Signier, D. A., Wang, H. P. (eds.) Development and application of Non-Newtonian flows,
ASME FED, Vol.231/MD Vol.66, pp.99-105 (1995).

[2] Masuda, H., Ebata, A., Teramae, K., Hishinuma, N.: Alteration of thermal conductivity and viscosity of liquid by dispersing ultra fine particles. Netsu Bussei 7, 227-233 (1993).

[3] Eastman, J. A., Choi, S. U. S., Tu, W., Thosmpson, L. J.: Anomalously increased effective thermal conductivities of ethylene glycol-based nanofluids containing copper nanoparticles. Appl. Phys. Lett. 78, 718-720(2001).

[4] Das, S. K., Putra, N., Thiesen, P., Roetzel, W.: Temperature dependence of thermal conductivity enhancement for nanofulids. ASME J. Heat Transf. 125, 567-574 (2003).

[5] Buongiorno, J.: Convective transport in nanofluids. ASME J. Heat Transer. 128, 240-250 (2006)

[6] Pearlstein, A. J: Effect of rotation on the stability of a doubly diffusive fluid layer. J. Fluid Mech. 103, 389-412(1981).

[7] Chakrabarti, A, Gupta, A. S.: Nonlinear thermo- haline convection in a rotating porous medium. Mech. Res. Commun. 8, 9-22(1981).

[8] Patil, P. R., Vidyanathan, G.: On setting up of convective currents in a ratoting porous medium under the influence of variable viscosity. Int. J. Eng. Sci. 21,123-130(1983).

[9] Vadasz, P: Free Convection in Rotating Porous Media, Transport Phenomena in Porous Media. pp. 285-312. Elsevier, Amsterdam (1998).

[10] Horton, W., Rogers, F. T.: Convection currents in a porous medium. J. Appl. Phys. 16, 367-370(1945).

[11] Lapwood, E. R.: Convection of a fluid in a porous medium. Proc. Camb. Phil. Soc. 44,508-521(1948).

[12] Neild, D. A.: Onset of thermohaline convection in a porous medium. Water Resour. Res. 4, 553-560(1968).

[13] Rudraiah, N., Malashetty, M. S.: The influence of couple molecular diffusion on the double diffusive convection in a porous medium. ASME J. Heat Transf. 108, 872-876(1986).

[14] Murray, B. T., Chen, C. F.: Double diffusive convection in a porous medium. J. Fluid Mech. 201, 147-166 (1989).

[15] Bhadauria, B. S.: Double diffusive convection in a porous medium with modulated temperature on the boundaries. Transp. Porous Med. 70, 191-211 (2007a).

[16] Vafai, K.: Handbook of Porous Media. Taylor and Francis, London (2005).

[17] Neild, D. A., Bejan, A.: Convection in Porous Media. 3rd edn. Springer, New York (2006).

[18] Neild, D. A., Kuznetsov, A. V.: Thermal instability in aporous medium layer saturated by nonofluid. Int. J. Heat Mass Transf. 52, 5796-5801(2009a).

[19] Neild, D. A., Kuznetsov, A. V.: The cheng-Minkowycz problem for natural convective boundary-layer flow in a porous medium saturated by a nanofluid. Int J. Heat Mass Transf. 52, 5792-5795(2009b).

[20] Neild, D. A., Kuznetsov, A. V.: The onset of convection in a horizontal nanofluid layer of finite dept. Eur. J. Mech. B 29 , 217-223 (2010). 
[21] Agarwal, S., Bhadauria, B. S., Siddheshwar, P. G.: Thermal instability of a nanofluid saturating a rotating anisotropic porous medium. Spec. Top. Rev. Porous Media Begell House Publ. 2(1), 53-64 (2001).

[22] Bhadauria, B. S., Agarwal, S., Kumar, A.: Non-linear twodimensional convection in a nanofluid saturated porous medium. Transp. Porous Media 90(2), 605-625(2011).

[23] Umavathi, J. C.: Rayleigh Benard convection subject to time dependent wall temperature in a porous medium layer saturated by a nanofluid. Meccanica. 50,981-984(2015)

[24] Umavathi, J. C. and Monica B Mohite.: Convective transport in a porous medium layer satuated with a Maxwell nanofluid. Jour of King Saud University-Engineering sciences. 28,5668(2016).

[25] Umavathi, J. C. and Prathap Kumar, J.: Onset of Convection in a porous medium layer saturated with an Oldoyd nanofluid, ASME J. Heat Transf. 139, 012401-0124014(2016).
[26] Nield, D. A., Bejan, A.: Convection in porous Medial, third ed., Springer, New York, 2006.

[27] Kuznetsov, A. V., Avramenko, A. A.: Effect of small particles on the stability of bioconvection in a suspension of gyrotactic microorganisms in a layerof finite length, Int. Commun. Heat Mass Transfer 31, 1-10 (2004).

[28] Buongiorno, J. and Hu, L. W. "Nanofluid Coolants for Advanced Nuclear Power Plants", Paper 5705, Proceedings of ICAPP '05, Seoul, May 15-19. (2005).

[29] Bhadauria B. S, Agarwal S: Natural convection in a nanofluid saturated rotating porous layer, a nonlinear study. Transp Porous Media 87(2), 585-602(2011).

[30] Tiwari R. K, Das M. K: Heat transfer augmentation in a twosided lid-driven differentially heated square cavity utilizing nanofluid. Int. J Heat Mass Transf, 50, 2002-2018(2007). 